\title{
Mikrobielle Nekromasse im Boden und deren Bedeutung für Bodenprozesse
}

ANJA MILTNER, MATTHIAS KÄSTNER

DEPARTMENT UMWELTBIOTECHNOLOGIE, HELMHOLTZ-ZENTRUM FÜR UMWELTFOR-

SCHUNG (UFZ), LEIPZIG

\section{Understanding soil organic matter (SOM) formation is crucial for sus- tainable land use and mitigation of global change. SOM is perceived nowadays as a continuum of plant and microbial residues at various stages of decay. Microbial residues play a dominant role; plant-derived matter is processed by microbes to biomass and finally to necromass stabilised in SOM. Carbon storage thus depends on microbial energy metabolism and on environmental factors and minerals of the respec- tive soils.}

DOI: $10.1007 / \mathrm{s} 12268-020-1351-0$

(c) Die Autoren 2020

Böden stellen die Basis für die Ernährung der gesamten Menschheit dar und müssen daher nachhaltig bewirtschaftet werden, um den steigenden Bedarf an Nahrungsmitteln

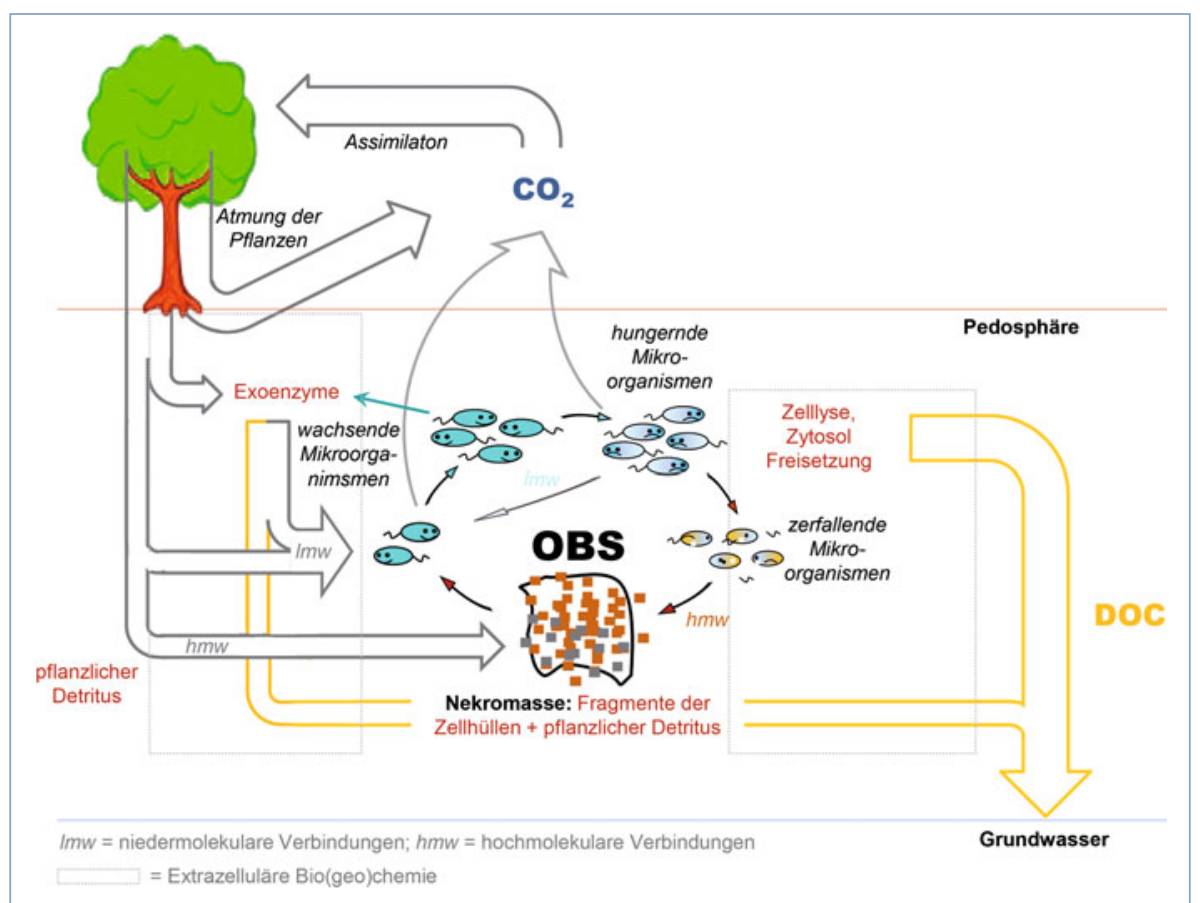

$\Delta$ Abb. 1: Prozesse, die die mikrobielle „Kohlenstoffpumpe“ im Boden mit Energie und Substraten versorgen und zur Stabilisierung bzw. Festlegung des Kohlenstoffs in der organischen Bodensubstanz (OBS) führen. Die „Kohlenstoffpumpe“ ist hier synonym mit dem mikrobiellen Wachstums- und Absterbezyklus. DOC: dissolved organic carbon. (Verändert nach [8].) telsicherheit. Die OBS stellt zudem eine wesentliche Senke für atmosphärischen Kohlenstoff (C) dar [1]; daher spielt der Erhalt der OBS-Gehalte eine Schlüsselrolle in der nachhaltigen Bodenbewirtschaftung und dem Klimaschutz. Dies wird im 4p1000-Programm zur Implementierung der OBS-Sequestrierung als nachhaltige Entwicklungsstrategie genutzt [2]. In der Landwirtschaft haben sich bekannte Methoden zum Erhalt der OBS, wie organische Dünger, das Mulchen mit Getreiderückständen oder der Anbau von Leguminosen bewährt, jedoch sind die Details der terrestrischen Stabilisierungsprozesse des Kohlenstoffs sowie deren mikrobiellen Steuerung nicht ausreichend verstanden [1]. Dies erschwert die effiziente Optimierung des Bodenmanagements; derzeit ist es noch nicht möglich, die Veränderungen der OBS-Gehalte unter verschiedenen Landnutzungen bei Änderungen der Umweltbedingungen (Klimawandel) sicher vorherzusagen, was aber für belastbare Modelle der $\mathrm{CO}_{2}$-Gehalte in der Atmosphäre nötig wäre.

Die OBS und die darin lebenden Mikroorganismen sind essenzielle Bestandteile des C-Kreislaufs auf der Erde. Die OBS stellt ein C-Reservoir „mittleren“ Oxidationsgrades zwischen der belebten Natur und Pflanzenwelt und der vollständigen Oxidation mit Rückführung des $\mathrm{CO}_{2}$ in die Atmosphäre dar. Neben den Bedingungen des jeweiligen Bodenstandortes hängt die Größe dieses Reservoirs vom zugeführten Material und der Energie aus der pflanzlichen Primärproduktion sowie vom Abbau und der Mineralisation durch die mikrobiellen Destruenten ab. Zunächst gelangt absterbendes Pflanzenmaterial in den Boden, welches deshalb lange Zeit auch als Ausgangsmaterial für die OBS angesehen wurde. Die Vorstellung bestand viele Jahre darin, dass sich während der mikrobiellen Zersetzung Abbauprodukte und schwer zersetzbare Bestandteile anreichern, welche dann entweder als solche oder durch Kondensationsreaktionen Huminstoffe bilden [3]. Daneben sollte ein wesentlich kleinerer Anteil mikrobieller Biomasse beim Abbau des Pflanzenmaterials entstehen, 
während große Mengen als $\mathrm{CO}_{2}$ freigesetzt werden. In der Tat liegt die gemessene Menge lebender mikrobieller Biomasse in einem engen Bereich bei zwei bis vier Prozent der OBS. Neuere Literatur zeigt jedoch einen wesentlich höheren Anteil an mikrobiellen Rückständen in der OBS als bisher aufgrund des kleinen Anteils lebender Biomasse angenommen wurde [1]. Abbaubares Pflanzenmaterial dient als Substrat für Mikroben, die darauf wachsen und sich vermehren. Dabei wird pflanzenbürtiger Kohlenstoff in mikrobielle Biomasse umgewandelt und der Rest entweder als $\mathrm{CO}_{2}$ in die Atmosphäre entlassen oder verbleibt als schwer abbaubarer Rest in der OBS (Abb. 1).

Da Mikroorganismen wegen ihrer Größe nur niedermolekulare Stoffe (<600 Da) aufnehmen können, müssen hochmolekulare pflanzenbürtige Stoffe wie Stärke, Cellulose, Lignin etc. zunächst durch Exoenzyme depolymerisiert werden, welche von den Mikroorganismen als „Investition in die Substratverfügbarkeit“ in ihre Umgebung abgegeben werden [3]. Dies führt zu einer komplexen Reaktivität in der OBS, die nicht im Innern der Zellen abläuft und post mortem zu bisher wenig untersuchten Modifikationen der Biomassebestandteile führen kann. Daher kann davon ausgegangen werden, dass nahezu alle Stoffe in der OBS von Mikroorganismen als Substrate genutzt werden, die irgendwie bioverfügbar sind. Allerdings können auch viele Stoffe vor einem Abbau geschützt sein, z. B. durch Wasserentzug, Sorption an Tonmineralen, Inkrustierung mit Metall- oder Silicium-Präzipitaten oder auch durch zelluläre Aggregation (z. B. Fragmente der Zellhüllen) [4].

Untersuchungen refraktärer organischer Substanz in marinen Systemen führte zur Entwicklung des Konzepts der microbial carbon pump als Teilprozess der biological pump. Es geht davon aus, dass biologischer Detritus und gelöste organische Verbindungen durch Mikroorganismen zersetzt und teilweise in mikrobielle Biomasse überführt werden [5]. Aus diesem Material wird dann partikuläre Nekromasse, welche in den marinen Sedimenten abgelagert wird und damit letztlich zum „Begraben“ des Kohlenstoffs im Sediment führt. Ein analoges Konzept wurde auch für Bodensysteme entworfen $[6,7]$, bei dem allerdings die Stabilisierung des Kohlenstoffs nicht durch „Begraben“ in Sedimenten erfolgt, sondern durch Schutz vor Abbau in den feinpartikulären Aggregaten.
Somit wird jede Verbindung, die bioverfügbar ist und genügend Energie beinhaltet, in mikrobielle Biomasse umgesetzt. Der mikrobielle Wachstums- und Absterbezyklus stellt damit die Überführung von leicht abbaubarem und verfügbarem pflanzlichen Kohlenstoff in mehr oder weniger refraktäre Nekromasse dar. Mit anderen Worten stammt der Kohlenstoff in der OBS ursprünglich aus pflanzlicher Biomasse, aus molekularer Sicht handelt es sich bei den OBS-Vorläufern aber um mikrobielle C-Verbindungen [8]. Erste Hinweise hierzu wurden in ${ }^{1} \mathrm{H}-\mathrm{NMR}-A$ nalysen von Huminsäuren aus Boden gefunden, in denen über 50 Prozent des NMR-Signals Komponenten mikrobiellen Ursprungs zugeordnet werden konnten [9]. Diese These wird durch den vielfach beobachteten langfristigen Verbleib (über ein Jahr) von ca. 50 Prozent des Kohlenstoffs aus mikrobieller Biomasse in der OBS gestützt, der mittels Inkubationsversuchen mit ${ }^{13} \mathrm{C}$-markierter bakterieller und pilzlicher Biomasse gezeigt wurde [4, 10]. In diesen Experimenten wurden die zugegebenen Zellen teilweise mineralisiert; je nach eingesetztem Organismus blieben bis zu 65 Prozent des markierten Kohlenstoffs im Boden zurück. Nur bis zu zehn Prozent des Kohlenstoffs wurden über die mikrobiellen Nahrungsnetze in lebende andere Organismen verteilt. Insgesamt wurden Phospholipidfettsäuren als Membranbestandteile wesentlich schneller abgebaut als der Gesamt-C aus der Biomasse, während Proteine erstaunlicherweise in der OBS stabilisiert und kaum mineralisiert wurden.

Die Überführung des pflanzlichen Biomasseeintrags in mikrobielle Bio- bzw. Nekromasse wird ebenso durch diverse Modellierungskonzepte untermauert $[4,11]$. Ein Teil der Nekromasse wird stabilisiert. Verfügbare Reste, die nach Absterben der Zellen und deren Zerfall entweder als Zellwandfragmente oder cytosolische Komponenten frei oder an Partikeloberflächen gebunden sind, können anderen Mikroorganismen wieder als Nährstoffe bzw. Bausteine für neue Wachstumszyklen dienen und erzeugen so fruchtbaren Boden. Kürzlich konnten wir mittels der gemessenen Konzentrationen von Aminozuckern aus den Resten mikrobieller Zellwände berechnen, wie groß der Anteil des C war, der als mikrobielle Nekromasse in der OBS an 122 unterschiedlichen Standorten festgelegt wurde. Je nach Landnutzungstyp betrug dieser Anteil 62 Prozent in Graslandstandorten, 56 Prozent in Ackerland und
33 Prozent in Waldstandorten [12]. Allerdings sind diese Zahlen nur dann wirklich so niedrig, wenn kein nennenswerter Abbau der Aminozucker stattfindet. Ansonsten stellen die gemessenen Konzentrationen nur das Fließgleichgewicht zwischen Bildung von mikrobieller Nekromasse und deren kontinuierlichem Abbau dar. In diesem (wahrscheinlichen) Fall müssen die Anteile des Mikroorganismen-bürtigen Kohlenstoffs noch deutlich höher sein.

Nicht nur Kohlenstoff, sondern auch ein hoher Anteil des in der OBS gebundenen Stickstoffs (N) ist mikrobiellen Ursprungs, wie das in Analysen von verschiedenen Standorten gezeigt werden konnte [13]. Das $\mathrm{C} / \mathrm{N}-$ Verhältnis in den feinen Bodenfraktionen $(<63 \mu \mathrm{m})$ ist mit etwa 10 dem Verhältnis von mikrobieller Biomasse von 5-7 wesentlich ähnlicher als dem von Pflanzenmaterial mit 20-80. Darüber hinaus zeigen NMRStudien [14], dass Stickstoff im Boden fast ausschließlich in Form von Amid-Stickstoff vorliegt, was auf Peptidbindungen, wie z. B. in Proteinen, hinweist, welche eigentlich als gut abbaubar gelten.

In Böden existieren zahlreiche Mechanismen zur Stabilisierung von organischem Material. Zunächst kann davon ausgegangen werden, dass die Überführung großer Mengen makromolekularer Pflanzeninhaltsstoffe in Fragmente der mikrobiellen Nekromasse bereits einen inhärenten Stabilisierungsmechanismus für dieses Material darstellt. Die Nekromassepartikel können sich zudem zu komplexen Aggregaten zusammenlagern, z. B. zu Stapeln aus Fragmenten der Zellhüllen [4], und schützen sich dabei gegenseitig vor enzymatischem Abbau. Auch eine Austrocknung solcher Aggregate führt aufgrund der hohen Lipidgehalte der Zellhüllen zu reduzierter Benetzbarkeit und damit einem verminderten mikrobiellen Abbau, da der mikrobielle Abbau generell von der lokalen chemischen Aktivität des Wassers abhängt. Weitere Mechanismen der Stabilisierung stellen die Sorption der Nekromassemoleküle und molekularen Aggregate an Mineraloberflächen bzw. der Einschluss in Minerale dar (z. B. Tone, Metalloxidhydrate, Carbonate etc.) [15], außerdem deren Inkrustierung mit Fällungsprodukten, z. B. mit Eisen- oder Manganoxiden, aufgrund von Redoxänderungen. Unter solchen Bedingungen in Böden und Sedimenten ist die mikrobielle Nekromasse daher metastabil und deren Inhaltsstoffe werden nach unseren Erfahrungen unterschiedlich stark stabilisiert: Proteine 
$>$ Peptidoglucane $>$ Polysaccharide $>$ Nukleinsäuren $>$ Lipide.

\section{Bedeutung für das Verständnis der Bodenprozesse und das Bodenmanagement}

Die Stabilisierung von Nekromasse in Böden erklärt auch, dass mikrobielle Biomoleküle und Fragmente mit zunehmender Tiefe relativ zunehmen, da hier der primäre Eintrag fehlt. Wenn zudem Fragmente von Zellhüllen und insbesondere Proteine im Boden stabilisiert werden, dann gilt dies vermutlich auch für Nukleinsäuren. Dies hätte wichtige Konsequenzen für metagenomische Konzepte zur Untersuchung mikrobieller Biozönosen in Böden [16]. Hier könnte bei Veränderungen der Umweltbedingungen die Besiedelungsgeschichte eine größere Diversität als die der tatsächlich lebenden Organismen vortäuschen.

Die Prozesse haben auch Auswirkungen auf das Verständnis diverser Eigenschaften und Funktionen von Böden:

- Die Speicherung von Kohlenstoff in der OBS hängt sowohl vom Energie- wie auch Nährstoffgehalt der zugeführten Substrate ab. Dies hat Konsequenzen für das Bodenmanagement in der Landwirtschaft und in der Bioökonomie.

- Mikrobielle Stoffwechselprozesse können modelliert und in die globalen Kohlenstoffumsatzmodelle mit eingebaut werden.

- Niedermolekulare Stoffe werden bevorzugt umgesetzt, da keine extrazelluläre Depolymerisation mehr nötig ist; daher ist die OBS arm an leicht abbaubaren niedermolekularen Stoffen.

- Hochmolekulare Stoffe (Stärke, Cellulose, Lignin, Proteine, Nukleinsäuren) müssen durch extrazelluläre Enzyme depolymerisiert werden, da sonst keine Aufnahme und kein Abbau durch Mikroorganismen möglich ist.

- Extrazelluläre Enzyme stellen daher „Investitionen“ der Organismen in die Verfügbarmachung von Substraten dar; allerdings erzeugen sie auch eine beträchtliche zellexterne Reaktivität.

- Die Mehrzahl der zugeführten Substrate wird in mikrobielle Biomasse und später Nekromasse umgesetzt.

- 30-50 Prozent des Kohlenstoffs aus der Biomasse verbleibt länger als ein Jahr in der OBS.

- Die OBS stellt das retardierte „Reservoir“ des Kohlenstoffumsatzes (inklusive Ener- giespeicherung) dar auf dem Weg vom Pflanzenmaterial zur Mineralisierung und der Freisetzung als $\mathrm{CO}_{2}$ in die Atmosphäre.

- Die relative Einheitlichkeit mikrobieller Biomasse mit vier verschiedenen Grundtypen (Gram-positive und Gram-negative Bakterien, Archaeen und Pilze) erklärt die Ähnlichkeit der OBS in verschiedenen Böden weltweit.

- Die Stabilisierung von Biomasse bzw. Nekromasse in der OBS erklärt:

- die Redoxeigenschaften aufgrund der Atmungskettenbestandteile der Fragmente der Zellhüllen;

- die Konservierung zellfreier Proteine und DNA-Moleküle;

- die Wasserspeicherung durch Schwellen und Schrumpfen von Fragmenten der Zellhüllen und Proteinrückständen;

- die Veränderungen der Priming-Effekte durch Nährstoffmobilisierung aus Nekromasse;

- die niedrigen C:N-Verhältnisse gegenüber Pflanzenmaterial;

- den Befund aus ${ }^{15} \mathrm{~N}-\mathrm{NMR}-A n a l y s e n$, dass nahezu 100 Prozent des Boden-N in Amidbindungen vorliegen;

- die Veränderung der Oberflächeneigenschaften von Mineralen, wie z. B. die Benetzbarkeit (Kontaktwinkel) durch Eigenschaften der Biomoleküle bzw. zelluläre Stressanpassung (veränderte Oberflächeneigenschaften und Inhaltsstoffe).

Das Wissen um die Prozesse der Bildung und Stabilisierung der OBS, insbesondere über die Rolle von mikrobieller Nekromasse, ermöglicht also, zielgerichtet Maßnahmen zur Erhaltung bzw. Erhöhung der OBS-Gehalte von Böden zu entwickeln, die gleichzeitig die Bodenfruchtbarkeit verbessern und dem Klimaschutz dienen.

\section{Danksagung}

Die Arbeiten wurden durch die DFG, die HGF, die EU sowie die Humboldt-Stiftung gefördert.

\section{Literatur}

[1] Schmidt MWI, Torn MS, Abiven S et al. (2011) Persistence of soil organic matter as an ecosystem property. Nature 478:49-56

[2] Rumpel C, Amiraslani F, Chenu C et al. (2020) The 4 p1000 initiative: opportunities, limitations and challenges for implementing soil organic carbon sequestration as a sustainable development strategy. Ambio 49:350-360

[3] Lehmann J, Kleber M (2015) The contentious nature of soil organic matter. Nature 528:60-68
[4] Miltner A, Bombach P, Schmidt-Brücken B et al. (2012 SOM genesis - microbial biomass as a significant source. Biogeochemistry 111:41-55

[5] Jiao N, Herndl GJ, Hansell DA et al. (2010) Microbial production of recalcitrant dissolved organic matter: long-term carbon storage in the global ocean. Nat Rev Microbiol 8:593599

[6] Fan Z, Liang C (2015) Significance of microbial asynchronous anabolism to soil carbon dynamics driven by litter inputs. Sci Rep 5:9575

[7] Liang C, Schimel JP, Jastrow JD (2017) The importance of anabolism in microbial control over soil carbon storage. Nat Microbiol 2:17105

[8] Kästner M, Miltner A (2018) SOM and microbes - what is left from microbial life. In: Garcia C, Nannipieri P, Hernandez $\mathrm{T}$ (Hrsg) The Future of Soil Carbon - Its conservation and formation. Elsevier Academic Press, New York. S 125-164

[9] Simpson AJ, Simpson MJ, Smith E et al. (2007) Microbially derived inputs to soil organic matter: are current estimates too low? Environ Sci Technol 41:8070-8076

[10] Schweigert M, Herrmann S, Miltner A et al. (2015) Fate of ectomycorrhizal fungal biomass in a soil bioreactor system and its contribution to soil organic matter formation. Soil Biol Biochem 88:120-127

[11] Liang C, Cheng G, Wixon D et al. (2011) An absorbing Markov chain approach to understanding the microbial role in soil carbon stabilization. Biogeochemistry 106:303-309 [12] Liang C, Amelung W, Lehmann J et al. (2019)

Quantitative assessment of microbial necromass contribution to soil organic matter. Glob Change Biol 25:3578-3590 [13] Liang C, Amelung W, Lehmann J et al. (2019) Quantitative assessment of microbial necromass contribution to soil organic matter. Glob Change Biol 25:3578-3590

[14] Knicker H (2011) Soil organic N - an underrated player for C sequestration in soils? Soil Biol Biochem 43:1118-1129 [15] Mikutta R, Schaumann GE, Gildemeister D et al. (2009) Biogeochemistry of mineral-organic associations across a long-term mineralogical soil gradient (0.3-4100 kyr), Hawaiian Islands. Geochim Cosmochim Acta 73:2034-2060 [16] Carini P, Marsden PJ, Leff JW et al. (2016) Relic DNA is abundant in soil and obscures estimates of soil microbial diversity. Nat Microbiol 2:16242

\section{Funding: Open Access funding provided by Projekt DEAL.}

Open Access: Dieser Artikel wird unter der Creative Commons Namensnennun 4.0 International Lizenz veröffentlicht, welche die Nutzung, Vervielfältigung, Bearbeitung, Verbreitung und Wiedergabe in jeglichem Medium und Form erlaubt, sofern Sie den/die ursprünglichen Autor(en) und die Quelle ordnungsgemäß nennen, einen Link zur Creative Commons Lizenz beifügen un angeben, ob Anderungen vorgenommen wurden. Die in diesem Artikel
enthaltenen Bilder und sonstiges Drittmaterial unterliegen ebenfalls der genannten Creative Commons Lizenz, sofern sich aus der Abbildungslegende nichts anderes ergibt. Sofern das betreffende Material nicht unter der genannten Creative Commons Lizenz steht und die betreffende Handlung nicht nach gesetzlichen Vorschriften erlaubt ist, ist für die oben aufgeführten Weiterverwendungen des Materials die Einwilligung des jeweiligen Rechteinhabers einzuholen. Weitere Details zur Lizenz entnehmen Sie bitte der Lizenzinformation auf http://creativecommons.org/licenses/by/4.0/deed.de.

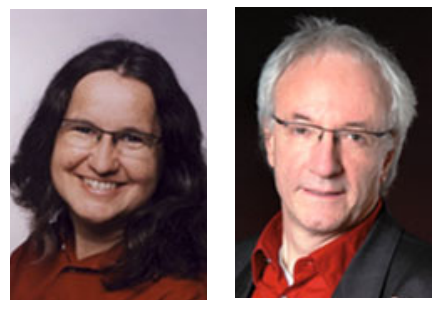

Anja Miltner und Matthias Kästner

Korrespondenzadresse:

Prof. Dr. habil. Matthias Kästner

Department Umweltbiotechnologie

Helmholtz-Zentrum für Umweltforschung (UFZ)

Permoserstraße 15

D-04318 Leipzig

matthias.kaestner@ufz.de

www.ufz.de/index.php?de $=2540$ 